\title{
RADEMACHER BOUNDED FAMILIES OF OPERATORS ON $L_{1}$
}

\author{
N. J. KALTON AND T. KUCHERENKO
}

(Communicated by N. Tomczak-Jaegermann)

\begin{abstract}
We give a characterization of R-bounded families of operators on $L_{1}$. We then use this result to study sectorial operators on $L_{1}$. We show that if $A$ is an R-sectorial operator on $L_{1}$, then, for any $\epsilon>0$, there is an invertible operator $U: L_{1} \rightarrow L_{1}$ with $\|U-I\|<\epsilon$ such that for some strictly positive Borel function $w, U(\mathcal{D}(A))$ contains the weighted $L_{1}$-space $L_{1}(w)$.
\end{abstract}

\section{INTRODUCTION}

Let us recall that a closed operator $A$ on a Banach space $X$ is called sectorial with sectoriality angle $\omega$ if

- The domain $\mathcal{D}(A)$ and range $\mathcal{R}(A)$ are dense

- $A$ is one-to-one

- The spectrum $\sigma(A)$ is contained in a closed sector $\Sigma_{\omega}=\{\zeta \in \mathbb{C}:|\arg \zeta| \leq$ $\omega\}$

- For any $\omega<\phi<\pi$ there is a constant $C_{\phi}$ such that the resolvent $R(\zeta, A)$ satisfies the estimate

$$
\|\zeta R(\zeta, A)\| \leq C_{\phi},|\arg (\zeta)| \geq \phi .
$$

Note that the definition does not require $A$ to be invertible. If $\omega<\frac{\pi}{2}$, then the operator $-A$ generates a bounded analytic semigroup, $T_{t}=e^{-t A}$. Conversely if $-A$ is the generator of a bounded analytic semigroup, then $A$ is sectorial with $\omega<\pi / 2$, provided it is one-one. For further discussion on sectorial operators see [2].

In applications involving $L_{p}$-maximal regularity of the abstract Cauchy problem or, more generally, the joint functional calculus of two commuting sectorial operators it is often important to know that a sectorial operator satisfies a stronger form of sectoriality, which we now introduce (see [8] and [11]).

We recall here that a collection of operators $\mathcal{T}$ on a Banach space $X$ is called $R$-bounded if there is a constant $C$ so that

$$
\left(\mathbb{E}\left\|\sum_{j=1}^{n} \epsilon_{j} T_{j} x_{j}\right\|^{2}\right)^{\frac{1}{2}} \leq C\left(\mathbb{E}\left\|\sum_{j=1}^{n} \epsilon_{j} x_{j}\right\|^{2}\right)^{\frac{1}{2}}, \quad x_{1}, \ldots, x_{n} \in X, T_{1}, \ldots, T_{n} \in \mathcal{T} .
$$

Received by the editors September 21, 2005 and, in revised form, December 6, 2006 and December 13, 2006.

2000 Mathematics Subject Classification. Primary 47D06, 46E30.

Key words and phrases. Sectorial operators, representation of regular operators, Rboundedness.

The authors acknowledge support from NSF grants DMS-0244515 and DMS-0555670. 
Here $\left(\epsilon_{j}\right)_{j=1}^{\infty}$ is a sequence of independent Rademacher functions. The KahaneKhintchine inequality allows us to replace the exponent 2 in the definition by any $p \geq 1$. $A$ is called $R$-sectorial with angle of R-sectoriality $\omega_{R}=\omega_{R}(A)$ if for every $\phi>\omega_{R}$ the collection of operators $\{\zeta R(\zeta, A):|\arg \zeta| \geq \phi\}$ is R-bounded.

If, for example, $A$ is invertible, then $R$-sectoriality with $\omega_{R}(A)<\pi / 2$ is necessary for $L_{p}$-maximal regularity of the abstract Cauchy problem $(1<p<\infty)$; if further $X$ is a UMD-space, it is also sufficient (see Weis [11] for details). Note that in a Hilbert space every sectorial operator is R-sectorial for the same angle.

This note is concerned with the structure of R-sectorial operators on the Banach space $L_{1}=L_{1}(K, \lambda)$ where $K$ is a Polish space (i.e. a topological space which is homeomorphic to a separable complete metric space) and $\lambda$ is a nonatomic $\sigma$-finite Borel measure. All such spaces are isometric to $L_{1}=L_{1}[0,1]$, and so we will assume that $K$ is a compact metric space and $\lambda$ is a probability measure.

Our work is related to some previous results which suggest that it is rather restrictive for a sectorial operator $A$ on $L_{1}$ to be R-sectorial. If $A$ is a sectorial operator on $L_{1}$ which has $H^{\infty}$-calculus (for some angle $\omega$ ), then $A$ is R-sectorial (for the same angle $\omega$ ) [8]. We refer to [8] for the definition and discussion of the $H^{\infty}$-calculus. In [8] it was shown that if $A$ has an $H^{\infty}$-calculus, then $A$ is bounded on any reflexive subspace of $\mathcal{D}(A)$ (with the graph norm); this had the implication that there are very few examples of sectorial operators with an $H^{\infty}$-calculus on $L_{1}$ and, in particular, essentially no reasonable differential operator can have this property. In [5] it was shown that there are no R-bounded strongly continuous semigroups on $L_{1}$ consisting of weakly compact operators; it also follows from the results of [5] that if $A$ is an $\mathrm{R}$-sectorial operator on $L_{1}$, then the resolvent $R(\zeta, A)$ can never be a weakly compact operator.

The simplest example of a sectorial operator on $L_{1}(K, \lambda)$ which has an $H^{\infty}$ calculus and hence is R-sectorial is the following. Given an a.e. positive function $b$ we define the operator

$$
A f(s)=b(s) f(s)
$$

with domain

$$
\mathcal{D}(A)=\left\{f: \int|f(s)| b(s)^{-1} d \lambda(s)<\infty\right\} .
$$

Note here that the domain is very large indeed; in fact for any $\epsilon>0$ we can find a Borel set $B$ with $\lambda(B)>1-\epsilon$ and such that $L_{1}(B) \subset \mathcal{D}(A)$. Of course one can get further examples by considering $A^{\prime}=U A U^{-1}$ for $U$ any invertible operator with $\mathcal{D}\left(A^{\prime}\right)=U(\mathcal{D}(A))$.

In this note, we show that this example is typical. Precisely, we show that if $A$ is R-sectorial and $\epsilon>0$, then there is an invertible operator $U: L_{1} \rightarrow L_{1}$ with $\|U-I\|<\epsilon$ such that for some positive Borel function $w$ we have $U(\mathcal{D}(A)) \supset L_{1}(w)$. This refines both the results of [5] and [8].

\section{Operators ON $L_{1}$}

Let $K$ be a compact metric space and suppose $\lambda$ is a probability measure on $K$. We denote by $\mathcal{B}(K)$ the $\sigma$-algebra of Borel sets on $K$ and by $\mathcal{M}(K)$ the space of Borel measures on $K$ with the norm of total variation. We will utilize the so-called random measure representation of operators on $L_{1}$, developed in [6], [4] and [10]. 
A random measure on $K$ is a map $s \rightarrow \mu_{s}$ from $K$ into $\mathcal{M}(K)$ which is Borel for the weak*-topology on $\mathcal{M}(K)$. If the random measure satisfies the condition

$$
\int_{K}\left|\mu_{s}\right|(B) d \lambda(s) \leq C \lambda(B), \quad B \in \mathcal{B}(K),
$$

then it induces a bounded operator $T: L_{1}(\lambda) \rightarrow L_{1}(\lambda)$ given by the formula

$$
T f(s)=\int_{K} f(t) d \mu_{s}(t) \quad \lambda-\text { a.e. }
$$

and then $\|T\| \leq C$.

Conversely every bounded linear operator $T: L_{1}(\lambda) \rightarrow L_{1}(\lambda)$ has an essentially unique random measure representation $s \rightarrow \mu_{s}^{T}$ and $\|T\|$ is the least constant $C$ so that (2.1) holds for $\mu_{s}^{T}$.

We may also associate to $T$ a unique measure $\rho_{T}$ on $K \times K$ given by

$$
\rho_{T}(E)=\int_{K}\left(\int_{K} \chi_{E}(s, t) d \mu_{s}^{T}(t)\right) d \lambda(s), \quad E \in \mathcal{B}(K \times K) .
$$

Thus

$$
\rho_{T}(A \times B)=\int_{A} T \chi_{B} d \lambda
$$

The map $T \rightarrow \rho_{T}$ maps the space of all bounded operators on $L_{1}(K)$, denoted by $\mathcal{L}\left(L_{1}\right)$, onto an order-ideal in $\mathcal{M}(K \times K)$ consisting of all measures $\rho$ such that

$$
|\rho|(A \times B) \leq C \lambda(B), \quad A, B \in \mathcal{B}(K \times K) .
$$

The space $\mathcal{L}\left(L_{1}(K, \lambda)\right)$ is a complex Banach lattice and it is easily checked that if $T \in \mathcal{L}\left(L_{1}\right)$, then $\mu_{s}^{|T|}=\left|\mu_{s}^{T}\right|$ ( $\lambda$-a.e.) and that $\rho_{|T|}=\left|\rho_{T}\right|$. Since it is a Banach lattice we can define as usual, using the Krivine calculus, an operator $\left(\sum_{j=1}^{n}\left|T_{j}\right|^{2}\right)^{\frac{1}{2}}$ for any $T_{1}, \ldots, T_{n} \in \mathcal{L}\left(L_{1}\right)$ (a full description of this construction is given in [9]).

The following result is implicitly contained in ideas of [6], and more explicitly in [7].

Proposition 2.1. Let $T_{n}: L_{1} \rightarrow L_{1}$ be a uniformly bounded sequence of operators such that $\lim _{n \rightarrow \infty}\left\|\rho_{T_{n}}\right\|=0$. Then given any $\epsilon>0$ there is a Borel subset $B$ of $K$ with $\lambda(B)>1-\epsilon$ and $n \in \mathbb{N}$ so that we have

$$
\left\|T_{n} f\right\| \leq \epsilon\|f\|, \quad f \in L_{1}(B) .
$$

Proof. Let $\sigma_{n}=\left|\rho_{T_{n}}\right|$. Consider the measure $\nu_{n}$ on $K$ given, for $A$ Borel, by

$$
\nu_{n}(A)=\sigma_{n}(A \times K)=\left\|\left|T_{n}\right| \chi_{A}\right\| .
$$

Then $\nu_{n}$ is absolutely continuous with respect to $\lambda$. Let $w_{n}$ be its Radon-Nikodym derivative. Then, by our hypothesis,

$$
\int w_{n} d \lambda=\sigma_{n}(K \times K) \rightarrow 0 .
$$

Therefore, $w_{n} \longrightarrow 0$ in measure. Hence there exists $n \in \mathbb{N}$ and $B$ with $\lambda(B)>1-\epsilon$ so that $\left|w_{n}\right|<\epsilon$ on $B$.

If $f \in L_{1}(B)$ we have

$$
\left\|T_{n} f\right\| \leq \int_{K \times K}|f(s)| d \sigma_{n}(s, t)=\int_{B}|f(s)| w_{n}(s) d \lambda(s) \leq \epsilon\|f\| .
$$


If $T \in \mathcal{L}\left(L_{1}\right)$, then we can write $\mu_{s}$ as given in $(2.2)$ in the form

$$
\mu_{s}=a(s) \delta_{s}+\mu_{s}^{\prime} \quad \lambda-\text { a.e. }
$$

where $\mu_{s}^{\prime}\{s\}=0 \lambda$-a.e. and $a$ is a bounded Borel function. (See for example [6].) Thus

$$
T f(s)=a(s) f(s)+\int_{K} f(t) d \mu_{s}^{\prime}(t) \quad \lambda-\text { a.e. }
$$

If we define the diagonal part of $T$ by

$$
\Pi(T) f=a(s) f(s),
$$

then $\rho_{\Pi(T)}$ is the restriction of $\rho_{T}$ to the diagonal subset $\Delta=\{(s, s): s \in K\}$. Thus

$$
\rho_{\Pi(T)}(B)=\rho_{T}(B \cap \Delta) .
$$

Theorem 2.2. Let $\mathcal{T}$ be a family of operators in $\mathcal{L}\left(L_{1}(K, \lambda)\right)$. Then the following are equivalent:

(i) $\mathcal{T}$ is $R$-bounded.

(ii) $\left\{\left(\sum_{k=1}^{n} a_{k}^{2}\left|T_{k}\right|^{2}\right)^{\frac{1}{2}}: \sum_{k=1}^{n}\left|a_{k}\right|^{2} \leq 1, T_{1}, \ldots, T_{n} \in \mathcal{T}, n \in \mathbb{N}\right\}$ is uniformly bounded.

Proof. Assume $\mathcal{T}$ is R-bounded, with

$$
\mathbb{E}\left\|\sum_{k=1}^{n} \epsilon_{k} T_{k} x_{k}\right\| \leq C \mathbb{E}\left\|\sum_{k=1}^{n} \epsilon_{k} x_{k}\right\|
$$

for any $T_{1}, \ldots, T_{n} \in \mathcal{T}$ and $x_{1}, \ldots, x_{n} \in X$. Suppose $T_{1}, \ldots, T_{n} \in \mathcal{T}$ and $a_{1}, \ldots, a_{n}$ $\in \mathbb{C}$ are such that $\sum_{k=1}^{n}\left|a_{k}\right|^{2} \leq 1$. Then, by Khintchine's inequality for lattices,

$$
\left\|\left(\sum_{k=1}^{n}\left|a_{k}\right|^{2}\left|T_{k}\right|^{2}\right)^{\frac{1}{2}}\right\| \leq M \mathbb{E}\left\|\left|\sum_{k=1}^{n} \epsilon_{k} a_{k} T_{k}\right|\right\|
$$

where $M$ is an absolute constant. Choose any sequence of partitions $\mathcal{A}_{m}=\left(A_{m j}\right)_{j=1}^{N_{m}}$ of $K$ so that each $\mathcal{A}_{m+1}$ refines $\mathcal{A}_{m}$ and

$$
\lim _{m \rightarrow \infty} \sup _{1 \leq j \leq N_{m}} \operatorname{diam} A_{m j}=0 .
$$

Then for any positive function $f \in L_{1}(K, \lambda)$ and any $T \in \mathcal{L}\left(L_{1}(K, \lambda)\right)$ we have

$$
|T| f=\lim _{m \rightarrow \infty} \sum_{j=1}^{N_{m}}\left|T\left(f \chi_{A_{m j}}\right)\right| \quad \lambda \text { - a.e. }
$$

Thus, replacing $T$ by $\sum_{k=1}^{n} \epsilon_{k} a_{k} T_{k}$ in the previous line yields

$$
\left|\sum_{k=1}^{n} \epsilon_{k} a_{k} T_{k}\right| f=\lim _{m \rightarrow \infty} \sum_{j=1}^{N_{m}}\left|\sum_{k=1}^{n} \epsilon_{k} a_{k} T_{k}\left(f \chi_{A_{m j}}\right)\right| \quad \lambda \text { - a.e. }
$$


Now, by R-boundedness

$$
\begin{aligned}
\mathbb{E} \int_{K} \sum_{j=1}^{N_{m}}\left|\sum_{k=1}^{n} \epsilon_{k} a_{k} T_{k}\left(f \chi_{A_{m j}}\right)\right| d \lambda & =\sum_{j=1}^{N_{m}} \mathbb{E}\left\|\sum_{k=1}^{n} \epsilon_{k} a_{k} T_{k}\left(f \chi_{A_{m j}}\right)\right\| \\
& \leq C \sum_{j=1}^{N_{m}} \mathbb{E}\left\|\sum_{k=1}^{n} \epsilon_{k} a_{k} f \chi_{A_{m j}}\right\| \\
& =C \sum_{j=1}^{N_{m}}\left\|f \chi_{A_{m} j}\right\| \mathbb{E}\left|\sum_{k=1}^{n} \epsilon_{k} a_{k}\right| \\
& =C\left(\sum_{k=1}^{n}\left|a_{k}\right|^{2}\right)^{\frac{1}{2}} \sum_{j=1}^{N_{m}}\left\|f \chi_{A_{m j}}\right\| \\
& \leq C\|f\|_{L_{1}} .
\end{aligned}
$$

It follows from Fatou's Lemma that

$$
\mathbb{E}\left\|\left|\sum_{k=1}^{n} \epsilon_{k} a_{k} T_{k}\right|\right\| \leq C
$$

and hence

$$
\left\|\left(\sum_{k=1}^{n}\left|a_{k}\right|^{2}\left|T_{k}\right|^{2}\right)^{\frac{1}{2}}\right\| \leq C M .
$$

We now prove that (ii) implies (i). First suppose $f \in L_{1}(K, \lambda)$ is positive and $T_{1}, \ldots, T_{n} \in \mathcal{L}\left(L_{1}(K, \lambda)\right)$. Then if $a_{1}, \ldots, a_{n} \geq 0$ and $a_{1}^{2}+\cdots+a_{n}^{2}=1$ we have

$$
\sum_{k=1}^{n} a_{k}\left|T_{k}\right| f \leq\left(\sum_{k=1}^{n}\left|T_{k}\right|^{2}\right)^{\frac{1}{2}} f
$$

The least upper bound of the left hand side over all choices of $a_{1}, \ldots, a_{n}$ is $\left(\sum_{k=1}^{n}\left(\left|T_{k}\right| f\right)^{2}\right)^{\frac{1}{2}}$ and so

$$
\left(\sum_{k=1}^{n}\left(\left|T_{k}\right| f\right)^{2}\right)^{\frac{1}{2}} \leq\left(\sum_{k=1}^{n}\left|T_{k}\right|^{2}\right)^{\frac{1}{2}} f
$$

Let us suppose $C$ is a constant so that

$$
\left\|\left(\sum_{k=1}^{n}\left|a_{k}\right|^{2}\left|T_{k}\right|^{2}\right)^{\frac{1}{2}}\right\| \leq C, \quad T_{1}, \ldots, T_{n} \in \mathcal{T},\left|a_{1}\right|^{2}+\cdots+\left|a_{n}\right|^{2}=1 .
$$

Suppose $f \in L_{1}$ and $T_{1}, \ldots, T_{n} \in \mathcal{T}$. Then

$$
\begin{aligned}
\mathbb{E}\left\|\sum_{k=1}^{n} \epsilon_{k} a_{k} T_{k} f\right\| & \leq\left\|\left(\sum_{k=1}^{n}\left|a_{k}\right|^{2}\left|T_{k} f\right|^{2}\right)^{\frac{1}{2}}\right\| \\
& \leq\left\|\left(\sum_{k=1}^{n}\left|a_{k}\right|^{2}\left(\left|T_{k}\right||f|\right)^{2}\right)^{\frac{1}{2}}\right\| \\
& \leq\left\|\left(\sum_{k=1}^{n}\left|a_{k}\right|^{2}\left|T_{k}\right|^{2}\right)^{\frac{1}{2}}|f|\right\| \\
& \leq C\|f\| .
\end{aligned}
$$

In this situation, Theorem 2.2 of [5] implies that $\mathcal{T}$ is R-bounded. 
Proposition 2.3. Suppose $\mathcal{T}$ is an R-bounded family of operators on $L_{1}(K, \lambda)$. Then the family of measures $\left\{\rho_{T}: T \in \mathcal{T}\right\}$ is relatively weakly compact in $\mathcal{M}(K \times K)$.

Proof. Let

$$
C=\sup \left\{\left\|\left(\sum_{j=1}^{m}\left|a_{j}\right|^{2}\left|T_{j}\right|^{2}\right)^{\frac{1}{2}}\right\|: T_{1}, \ldots, T_{m} \in \mathcal{T}, \sum_{j=1}^{m}\left|a_{j}\right|^{2} \leq 1, m \in \mathbb{N}\right\},
$$

which is finite by Theorem 2.2. Now, if $T_{1}, \ldots, T_{n} \in \mathcal{T}$, then

$$
\left\|\max _{1 \leq k \leq n}\left|T_{k}\right|\right\| \leq\left\|\left(\sum_{k=1}^{n}\left|T_{k}\right|^{2}\right)^{\frac{1}{2}}\right\| \leq C n^{\frac{1}{2}}
$$

The maximum here is computed in the lattice $\mathcal{L}\left(L_{1}\right)$.

Hence

$$
\left\|\max _{1 \leq k \leq n}\left|\rho_{T_{k}}\right|\right\|_{\mathcal{M}(K \times K)} \leq C n^{\frac{1}{2}} .
$$

Assume the set $\left\{\rho_{T}: T \in \mathcal{T}\right\}$ is not relatively weakly compact. Then there is a $\delta>0$, a sequence $\left(T_{k}\right)_{k=1}^{n}$ and a sequence of disjoint open sets $U_{k}$ in $K \times K$ so that $\rho_{T_{k}}\left(U_{k}\right) \geq \delta$ for all $k$ (see e.g. [3]). Then

$$
\left\|\max _{1 \leq k \leq n}\left|\rho_{T_{k}}\right|\right\|_{\mathcal{M}(K \times K)} \geq \sum_{k=1}^{n} \rho_{T_{k}}\left(U_{k}\right) \geq \delta n, \quad n=1,2, \ldots,
$$

which gives a contradiction.

\section{Applications to sectorial operators}

In this section we give some applications of the above results to sectorial operators.

Proposition 3.1. If $A$ is $R$-sectorial and $\omega_{R}(A)<\pi / 2$, then $\left\{e^{-t A}: 0<t<\infty\right\}$ is an $R$-bounded semigroup. Conversely, if $A$ is sectorial and $-A$ generates an $R$-bounded semigroup, then $A$ is $R$-sectorial with $\omega_{R}(A) \leq \pi / 2$.

If $-A$ is a sectorial operator which generates a semigroup $\left\{e^{-t A}: 0<t<\infty\right\}$ with the property that $\left\{e^{-t A}: 0<t \leq 1\right\}$ is $R$-bounded, then for any $\phi>\pi / 2$ there exists $M$ so that the set $\{\zeta R(\zeta, A):|\arg (\zeta+M)| \geq \phi\}$ is $R$-bounded.

Proof. Our proof depends mainly on the two formulas

$$
\zeta R(\zeta, A)=\int_{0}^{\infty} \zeta e^{\zeta t} e^{-t A} d t
$$

and

$$
e^{-t A}-(1+t A)^{-1}=-\frac{1}{2 \pi i} \int_{\Gamma_{\nu}}\left(e^{-t \zeta}-(1+t \zeta)^{-1}\right) R(\zeta, A) d \zeta
$$

where $\Gamma_{\nu}$ is a contour of the form $\left\{|s| e^{i(\operatorname{sgn} s) \nu}:-\infty<s<\infty\right\}$ for any $\nu$ with $\nu>\omega(A)$. 
Assuming that $\left\{e^{-A t}: 0<t<\infty\right\}$ is R-bounded we fix some angle $\frac{\pi}{2}<\varphi<\pi$. Then for any choice of numbers $\zeta_{j}=r_{j} e^{i \varphi_{j}}$ with $\varphi_{j} \geq \varphi, j=1, \ldots, n$, we obtain

$$
\begin{aligned}
\mathbb{E}\left\|\sum_{j=1}^{n} \epsilon_{j} \zeta_{j} R\left(\zeta_{j}, A\right) x_{j}\right\| & =\mathbb{E}\left\|\sum_{j=1}^{n} \int_{0}^{\infty} \epsilon_{j} r_{j} e^{i \varphi_{j}} e^{t r_{j} e^{i \varphi_{j}}} e^{-t A} x_{j} d t\right\| \\
& =\mathbb{E}\left\|\sum_{j=1}^{n} \int_{0}^{\infty} \epsilon_{j} e^{i \varphi_{j}} e^{s e^{i \varphi_{j}}} e^{-s / r_{j} A} x_{j} d s\right\| \\
& \leq \int_{0}^{\infty} \mathbb{E}\left\|\sum_{j=1}^{n} \epsilon_{j} e^{i \varphi_{j}} e^{s e^{i \varphi_{j}}} e^{-s / r_{j} A} x_{j}\right\| d s \\
& \leq C \int_{0}^{\infty} \max _{j}\left|e^{s e^{i \varphi_{j}}}\right| d s \cdot \mathbb{E}\left\|\sum_{j=1}^{n} \epsilon_{j} x_{j}\right\| \\
& \leq C \int_{0}^{\infty} e^{s \cos \varphi} d s \cdot \mathbb{E}\left\|\sum_{j=1}^{n} \epsilon_{j} x_{j}\right\| \\
& \leq \frac{C}{|\cos \varphi|} \cdot \mathbb{E}\left\|\sum_{j=1}^{n} \epsilon_{j} x_{j}\right\| .
\end{aligned}
$$

Therefore $A$ is R-sectorial with sectoriality angle $\omega_{R}(A) \leq \pi / 2$. Similarly, it follows that if $A$ is R-sectorial and $\omega_{R}(A)<\pi / 2$, then $\left\{e^{-t A}: 0<t<\infty\right\}$ is R-bounded.

For the last statement suppose that $C$ is a constant such that

$$
\left(\mathbb{E}\left\|\sum_{j=1}^{n} \epsilon_{j} e^{-t_{j} A} x_{j}\right\|^{2}\right)^{\frac{1}{2}} \leq C\left(\mathbb{E}\left\|\sum_{j=1}^{n} \epsilon_{j} x_{j}\right\|^{2}\right)^{\frac{1}{2}}
$$

whenever $x_{1}, \ldots, x_{n} \in X, 0 \leq t_{1}, \ldots, t_{n} \leq 1$.

Then if $m \in \mathbb{N}$,

$$
\left(\mathbb{E}\left\|\sum_{j=1}^{n} \epsilon_{j} e^{-\left(m+t_{j}\right) A} x_{j}\right\|^{2}\right)^{\frac{1}{2}} \leq C K^{m}\left(\mathbb{E}\left\|\sum_{j=1}^{n} \epsilon_{j} x_{j}\right\|^{2}\right)^{\frac{1}{2}}
$$

where $K=\left\|e^{-A}\right\|$. Now we show that the set $\left\{e^{-u t} e^{-t A}: 0<t<\infty\right\}$ is R-bounded as long as $e^{u}>K$. For $x_{1}, \ldots, x_{n} \in X$ and $0<t_{1}, \ldots, t_{n}<\infty$ we obtain

$$
\begin{aligned}
\left(\mathbb{E}\left\|\sum_{j=1}^{n} \epsilon_{j} e^{-t_{j} u} e^{-t_{j} A} x_{j}\right\|^{2}\right)^{\frac{1}{2}} & =\left(\mathbb{E}\left\|\sum_{m=0}^{\infty} \sum_{m \leq t_{j}<m+1} \epsilon_{j} e^{-t_{j} u} e^{-t_{j} A} x_{j}\right\|^{2}\right)^{\frac{1}{2}} \\
& \leq C \sum_{m=0}^{\infty} K^{m} e^{-u m}\left(\mathbb{E}\left\|\sum_{m \leq t_{j}<m+1} \epsilon_{j} e^{-u \tilde{t}_{j}} x_{j}\right\|^{2}\right)^{\frac{1}{2}}
\end{aligned}
$$

where $0 \leq \tilde{t}_{j} \leq 1$. By the contraction principle

$$
\left(\mathbb{E}\left\|\sum_{m \leq t_{j}<m+1} \epsilon_{j} e^{-u \tilde{t_{j}}} x_{j}\right\|^{2}\right)^{\frac{1}{2}} \leq \max _{1 \leq j \leq n}\left|e^{-u \tilde{t_{j}}}\right|\left(\mathbb{E}\left\|\sum_{j=1}^{n} \epsilon_{j} x_{j}\right\|^{2}\right)^{\frac{1}{2}} \leq\left(\mathbb{E}\left\|\sum_{j=1}^{n} \epsilon_{j} x_{j}\right\|^{2}\right)^{\frac{1}{2}} .
$$

Since $\sum_{m=0}^{\infty} K^{m} e^{-u m}$ is finite for $u>\ln K$ we obtain the claim. Consequently, the set $\{\xi R(\xi, u+A):|\arg \xi|>\phi\}$ is R-bounded. 
Now for $\zeta \in \mathbb{C}$ with $|\arg (\zeta+M)|>\phi, M>u$, we can rewrite using $\xi-u=\zeta$,

$$
\zeta R(\zeta, A)=(\xi-u) R(\xi-u)=(\xi-u) R(\xi, A+u)=\frac{\xi-u}{\xi} \xi R(\xi, A+u) .
$$

Since $\left|\frac{\xi-u}{\xi}\right| \leq \frac{M}{M-u}$ the result follows quickly.

It follows from results of [5] that if $-A$ is the generator of a semigroup such that $e^{-t A}$ is weakly compact for $t>0$, or if the resolvents $R(z, A)$ are weakly compact operators, then $A$ cannot be $\mathrm{R}$-sectorial. The next theorem strengthens this conclusion.

Theorem 3.2. Suppose $A$ is a sectorial operator on $L_{1}(K, \lambda)$. Assume that either:

(i) $A$ is $R$-sectorial for some angle $\omega$, or

(ii) $-A$ is the generator of a bounded semigroup such that $\left\{e^{-t A}: 0<t \leq 1\right\}$ is $R$-bounded.

Then there is a bounded function $a(\zeta, s)$ defined for $s \in K$ and $|\arg \zeta|>\omega$ such that

- For each $s \in K$ the $\operatorname{map} \zeta \rightarrow a(\zeta, s)$ is analytic.

- For each $\zeta$ the map $s \rightarrow a(\zeta, s)$ is Borel.

- $\lambda\{s: a(\zeta, s)=0\}=0$ for almost every $\zeta$.

$$
(R(\zeta, A) f)(s)=a(\zeta, s) f(s)+\int_{K} f(t) d \mu_{s}^{\zeta}(t), \quad f \in L_{1},
$$

where $\mu_{s}^{\zeta}\{s\}=0$.

Proof. We begin with the observation that, under either hypothesis, there exist $\phi<\pi$ and $M<\infty$ such that the set of operators $\{\zeta R(\zeta, A):|\arg \zeta| \geq \phi,|\zeta| \geq M\}$ is R-bounded. Hence the set of measures $\left\{\rho_{R(\zeta, A)}:|\arg \zeta| \geq \phi,|\zeta| \geq M\right\}$ is relatively weakly compact.

Consider the map $\zeta \rightarrow \Pi(R(\zeta, A))$ which is an analytic map from the set $\mathcal{S}=$ $\{\zeta:|\arg \zeta|>\omega\}$ into $\mathcal{L}\left(L_{1}\right)$. This induces an analytic map $F: \mathcal{S} \rightarrow L_{\infty}(K, \lambda)$ given by

$$
\Pi(R(\zeta, A)) f=F(\zeta) f .
$$

Let us show that we can choose representatives so that $F(\zeta)(s)=a(\zeta, s)$ where $a$ satisfies the first two conditions of the statement. Indeed let $\mathbb{D}$ be the unit disk and let $\varphi: \mathbb{D} \rightarrow \mathcal{S}$ be a conformal equivalence. Then $F \circ \phi$ can be expanded in a Taylor series around the origin and we may pick uniformly bounded Borel representatives $b_{n}$ for the coefficients in the expansion so that

$$
F(\varphi(z))(s)=\sum_{n=0}^{\infty} b_{n}(s) z^{n} \quad \lambda-\text { a.e., } z \in \mathbb{D} .
$$

Let

$$
a(\zeta, s)=\sum_{n=0}^{\infty} b_{n}(s)\left(\varphi^{-1}(\zeta)\right)^{n} .
$$

Assume that the third condition fails. Then by Fubini's theorem there is a subset $B$ of $K$ with $\lambda(B)>0$ so that for each $s \in B$ the set $\{\zeta: a(\zeta, s)=0\}$ has positive planar measure. By analyticity, this implies $a(\zeta, s) \equiv 0$ for $s \in B$.

However $\rho_{n(n+A)^{-1}}$ converges weakly to $\rho_{I}$ and hence so does $\rho_{\Pi\left(n(n+A)^{-1}\right)}$. Thus $-n a(-n, s)$ is weakly convergent to the constant function $1 \in L_{1}(K, \lambda)$. This is a contradiction. 
The next theorem shows that if a sectorial operator generates an R-bounded semigroup on $L_{1}$, then it is very similar to a bounded operator in the sense that its domain is sufficiently large to contain generic $L_{1}$-functions.

Theorem 3.3. Let $A$ be a sectorial operator on $L_{1}(K, \lambda)$ and assume that for some $\phi<\pi$ and $M<\infty$ the set $\{\zeta R(\zeta, A):|\arg \zeta| \geq \phi,|\zeta| \geq M\}$ is R-bounded. Then for any $\epsilon>0$ there is an invertible operator $U: L_{1} \rightarrow L_{1}$ with $\|U-I\|<\epsilon$ and a density function $w>0$ a.e. such that $L_{1}(w) \subset U^{-1}(\mathcal{D}(A))$.

In particular, there is a closed subspace $Y$ of $\mathcal{D}(A)$ isomorphic to $L_{1}$ so that $A: Y \rightarrow A(Y)$ is bounded (and thus $Y$ is also closed in $L_{1}$ ).

Proof. According to Proposition 2.3 the set of measures $\rho_{\zeta R(\zeta, A)}$ for $|\arg \zeta| \geq \phi,|\zeta|$ $\geq M$ is relatively weakly compact in $\mathcal{M}(K \times K)$. The sequence $\left(m(m+A)^{-1}\right)_{m \geq M}$ converges in the strong operator topology to the identity. Therefore, $\rho_{m(m+A)^{-1}}$ converges weak* to $\rho_{I}$ in $\mathcal{M}(K \times K)$ and hence converges weakly to $\rho_{I}$ by weak compactness.

Fix $\epsilon>0$. We may find a sequence of convex combinations $\left(T_{n}\right)_{n=1}^{\infty}$ of $\left\{m(m+A)^{-1}\right\}_{m=1}^{\infty}$ such that $\rho_{T_{n}}$ converges to $\rho_{I}$ in norm. Applying Proposition 2.1 to $\left(T_{n}-I\right)_{n=1}^{\infty}$ gives a sequence of Borel sets $E_{n} \subset K$ such that $\lambda\left(E_{n}\right)>1-2^{-n} \epsilon$ and

$$
\left\|T_{n} f-f\right\| \leq 2^{-n} \epsilon\|f\|, \quad f \in L_{1}\left(E_{n}\right) .
$$

Let us put $F_{1}=E_{1}$ and then $F_{n}=E_{n} \backslash E_{n-1}$ for $n \geq 2$. We define $U: L_{1} \rightarrow L_{1}$ by

$$
U f=\sum_{n=1}^{\infty} T_{n}\left(f \chi_{F_{n}}\right) .
$$

Thus $\|U-I\| \leq \epsilon$. Observe that $T_{n}: L_{1} \rightarrow \mathcal{D}(A)$ and so $A T_{n}$ is a bounded operator on $L_{1}$.

Define

$$
w=\sum_{n=1}^{\infty}\left\|A T_{n}\right\| \chi_{F_{n}}
$$

and assume $f \in L_{1}(w)$. Then

$$
\left\|A U\left(f \chi_{F_{n}}\right)\right\|=\left\|A T_{n}\left(f \chi_{F_{n}}\right)\right\| \leq \int_{F_{n}}|f| w d t .
$$

Hence $\sum_{k=1}^{\infty} A U\left(f \chi_{F_{k}}\right)$ converges and, since $A$ is closed, $U f \in \mathcal{D}(A)$.

The last part of the theorem is deduced by fixing any $n$ and note that if $Y=$ $U\left(L_{1}\left(E_{n}\right)\right)$, then $A$ is bounded on $Y$ and hence $Y$ is closed in both $\mathcal{D}(A)$ and $X$ and is isomorphic to $L_{1}$ in both.

Many differential operators on bounded domains have compact resolvents. Therefore we can use the results of [5] to show that they cannot be R-sectorial. In contrast, resolvents of differential operators on unbounded domains are, in general, not compact. An important example is the Laplacian $\Delta$ on $L_{1}\left(\mathbb{R}^{n}\right)$. Our corollary addresses this situation.

Corollary 3.4. Let $\Omega \subset \mathbb{R}^{n}$ be a bounded open set with locally Lipschitz boundary or $\Omega=\mathbb{R}^{n}$. Suppose that $A: \mathcal{D}(A) \subset L_{1}(\Omega) \longrightarrow L_{1}(\Omega)$ is a sectorial operator such that $\mathcal{D}(A)$ is contained in a Sobolev space $H_{1}^{s}(\Omega)$ for some $s>0$. Then $A$ does not generate an $R$-bounded semigroup. 
Proof. Assume the contrary, i.e., $A$ generates an R-bounded semigroup. Then by Sobolev's embedding theorem [1] we have a continuous inclusion $H_{1}^{s}(\Omega) \hookrightarrow$ $L_{p}(\Omega) \cap L_{1}(\Omega)$ for some $p>1$. By Theorem 3.3 there is a closed subspace $Y$ of $\mathcal{D}(A)$ on which $A$ is bounded and so that $Y$ is isomorphic to $L_{1}$. This implies that there is a subspace of $L_{1}(\Omega) \cap L_{p}(\Omega)$ which is isomorphic to $L_{1}$. If $\Omega$ is bounded this is an immediate contradiction since $L_{1}(\Omega) \cap L_{p}(\Omega)=L_{p}(\Omega)$ is reflexive. However even if $\Omega$ is unbounded this is still impossible. If $\Omega=\mathbb{R}^{n}$ we consider an isomorphism $J: L_{1} \rightarrow L_{1}\left(\mathbb{R}^{n}\right) \cap L_{p}\left(\mathbb{R}^{n}\right)$. Then $J: L_{1} \rightarrow L_{p}\left(\mathbb{R}^{n}\right)$ is a Dunford-Pettis operator and so if $\left(f_{n}\right)$ is any normalized weakly null sequence in $L_{1}$ we have $\left\|J f_{n}\right\|_{p} \rightarrow 0$. By passing to a subsequence we can assume $J f_{n} \rightarrow 0$ a.e. But then $\left(J f_{n}\right)$ is also weakly null in $L_{1}\left(\mathbb{R}^{n}\right)$ and so $\left\|J f_{n}\right\|_{1} \rightarrow 0$. This gives a contradiction.

This corollary is actually true for any set $\Omega$ for which Sobolev's embedding theorem holds. Sufficient geometrical properties of $\Omega$ for this to happen are discussed in $[1]$.

\section{REFERENCES}

[1] R. A. Adams, Sobolev spaces, Pure and Applied Mathematics, Vol. 65, Academic Press, New York-London, 1975. MR0450957 (56:9247)

[2] M. Cowling, I. Doust, A. McIntosh, and A. Yagi, Banach space operators with a bounded $H^{\infty}$ functional calculus, J. Austral. Math. Soc. Ser. A 60 (1996), 51-89. MR1364554 (97d:47023)

[3] J. Diestel, Sequences and series in Banach spaces, Graduate Texts in Mathematics, vol. 92, Springer-Verlag, New York, 1984. MR737004 (85i:46020)

[4] H. Fakhoury, Représentations d'opérateurs à valeurs dans $L^{1}(X, \Sigma, \mu)$, Math. Ann. 240 (1979), 203-212 (French). MR526843 (80e:47027)

[5] M. Hoffmann, N. Kalton, and T. Kucherenko, R-bounded approximating sequences and applications to semigroups, J. Math. Anal. Appl. 294 (2004), 373-386. MR2061331 (2005e:46034)

[6] N. J. Kalton, The endomorphisms of $L_{p}(0 \leq p \leq 1)$, Indiana Univ. Math. J. 27 (1978), 353-381. MR0470670 (57:10416)

[7] Linear operators on $L_{p}$ for $0<p<1$, Trans. Amer. Math. Soc. 259 (1980), 319-355. MR0567084 (81d:47022)

[8] N. J. Kalton and L. Weis, The $H^{\infty}$-calculus and sums of closed operators, Math. Ann. 321 (2001), 319-345. MR1866491 (2003a:47038)

[9] J. Lindenstrauss and L. Tzafriri, Classical Banach spaces, II, Function spaces, Vol. 97, Springer-Verlag, Berlin, 1979. MR540367 (81c:46001)

[10] L. Weis, On the representation of order continuous operators by random measures, Trans. Amer. Math. Soc. 285 (1984), 535-563. MR752490 (85g:47047)

[11] _ Operator-valued Fourier multiplier theorems and maximal $L_{p}$-regularity, Math. Ann. 319 (2001), 735-758. MR1825406 (2002c:42016)

Department of Mathematics, University of Missouri-Columbia, Columbia, Missouri 65211

E-mail address: nigel@math.missouri.edu

Department of Mathematics, University of California los Angeles, Box 951555, Los Angeles, California 90095-1555

E-mail address: tamara@math.ucla.edu 